\title{
Role of MTHFR C677T polymorphism in systemic carcinogenesis, besides its role in the pathogenesis of colo-rectal carcinomas
}

\author{
S. Kapoor \\ Mechanicsville, VA, USA \\ Corresponding author: S. Kapoor \\ E-mail: shailendrakapoor@yahoo.com \\ Genet. Mol. Res. 11 (3): 3365-3366 (2012) \\ Received July 27, 2012 \\ Accepted August 20, 2012 \\ Published September 24, 2012 \\ DOI http://dx.doi.org/10.4238/2012.September.24.2
}

Dear Editor,

I read with great interest the recent article by Sameer et al. (2011). Interestingly, the past few years have seen the emergence of evidence that the MTHFR C677T polymorphism contributes to systemic carcinogenesis in other organs, besides playing a role in the pathogenesis of colorectal cancer. For instance, an increased risk of esophageal cancer is seen in individuals with the MTHFR C677T polymorphism, especially those exhibiting the TT genotype (Liu et al., 2011). The carcinogenic effects of the MTHFR C677T polymorphism are enhanced in smokers and heavy ethanol consumers. Folic acid attenuates the carcinogenic risk in these individuals. Interestingly, synergism is seen with the CYP4502E1 gene. In fact, the MTHFR677 $(\mathrm{C} / \mathrm{T}+\mathrm{T} / \mathrm{T})$ and CYP4502E1C1/C1 genotypes increase the risk of developing esophageal cancer by almost seven times (Langevin et al., 2009).

Similarly, Zhang et al. (2010), in a recent meta-analysis have shown that the TT genotype is associated with accentuated risk of developing breast carcinomas. Similarly, the C677T polymorphism enhances the risk of early onset cervical carcinogenesis. In fact, in a recent report the odds ratio for developing cervical carcinoma for the MTHFR TT genotype was 1.4 in comparison with the MTHFR CC genotype (Sull et al., 2004). Interestingly, decreased risk for developing prostate cancer is seen in males with the CT genotype (Kucukhuseyin et al., 
2011). Similarly, the MTHFR C677T polymorphism exerts a slight protective effect against pulmonary carcinomas (Cui et al., 2011).

The above examples illustrate a close relationship between the MTHFR C677T polymorphism and systemic carcinogenesis. Further large-scale studies are needed to reaffirm and confirm these findings.

\section{REFERENCES}

Cui LH, Shin MH, Kim HN, Song HR, et al. (2011). Methylenetetrahydrofolate reductase C677T polymorphism in patients with lung cancer in a Korean population. BMC Med. Genet. 12: 28.

Kucukhuseyin O, Kurnaz O, Akadam-Teker AB, Narter F, et al. (2011). Effects of the MTHFR C677T polymorphism on prostate specific antigen and prostate cancer. Asian Pac. J. Cancer Prev. 12: 2275-2278.

Langevin SM, Lin D, Matsuo K, Gao CM, et al. (2009). Review and pooled analysis of studies on MTHFR C677T polymorphism and esophageal cancer. Toxicol. Lett. 184: 73-80.

Liu YX, Wang B, Wan MH, Tang WF, et al. (2011). Meta-analysis of the relationship between the Metholenetetrahydrofolate reductase C677T genetic polymorphism, folate intake and esophageal cancer. Asian Pac. J. Cancer Prev. 12: 247252.

Sameer AS, Shah ZA, Nissar S, Mudassar S, et al. (2011). Risk of colorectal cancer associated with the methylenetetrahydrofolate reductase (MTHFR) C677T polymorphism in the Kashmiri population. Genet. Mol. Res. 10: $1200-1210$

Sull JW, Jee SH, Yi S, Lee JE, et al. (2004). The effect of methylenetetrahydrofolate reductase polymorphism C677T on cervical cancer in Korean women. Gynecol. Oncol. 95: 557-563.

Zhang J, Qiu LX, Wang ZH, Wu XH, et al. (2010). MTHFR C677T polymorphism associated with breast cancer susceptibility: a meta-analysis involving 15,260 cases and 20,411 controls. Breast Cancer Res. Treat. 123: 549-555. 